\title{
Erectile Dysfunction and Cardiovascular Risk in Men With Rheumatoid Arthritis: A Population-based Cohort Study
}

\author{
Katelynn M. Wilton ${ }^{1}\left(\mathbb{D}\right.$, Sara J. Achenbach ${ }^{2}$, John M. Davis III ${ }^{3}$ (D), Elena Myasoedova ${ }^{4}$ (D), \\ Eric L. Matteson ${ }^{4}$, and Cynthia S. Crowson ${ }^{5}$ (D)
}

\begin{abstract}
Objective. Both erectile dysfunction (ED) and rheumatoid arthritis (RA) are associated with increased cardiovascular $(\mathrm{CV})$ risk. It is unknown if these diagnoses are associated or if their combination confers additional $\mathrm{CV}$ risk. We aimed to define the incidence of ED in RA, and to determine if ED correlates with increased CV risk in RA.

Methods. Medical information concerning RA, ED, and CV diagnoses for men with RA ( $\mathrm{n}=260)$ diagnosed in Olmsted County, Minnesota, and age-matched male comparators was extracted from a comprehensive medical record system.

Results. ED incidence was similar between the RA cohort and comparators (HR 0.80, 95\% CI 0.55-1.16). In men with RA, ED diagnosis was associated with a trend toward an increase in peripheral arterial disease (HR 2.22, 95\% CI 0.98-5.03) and a significantly decreased rate of myocardial infarction (HR 0.26, 95\% CI 0.07-0.90), heart failure (HR 0.49, 95\% CI 0.25-0.94), and death (HR 0.56; 95\% CI 0.36-0.87). In men with RA and ED, phosphodiesterase-5 inhibitor use was associated with a decreased risk of death (HR 0.35 , 95\% CI 0.16-0.79), with a trending decreased risk of some CV diagnoses.

Conclusion. Incidence of ED was not statistically increased in RA. Although patients with both RA and ED had a similar overall CV risk to those with RA alone, men with both RA and ED had decreased risk of heart failure, myocardial infarction, and death, as well as an increased risk of peripheral arterial disease. Further studies are needed to clarify these associations and their implications for pathogenesis and therapeutics.
\end{abstract}

Key Indexing Terms: cardiovascular risk, epidemiology, erectile dysfunction, peripheral arterial disease, rheumatoid arthritis, sexual health

KMW was supported by the National Institutes of General Medical Sciences (grant T32-GM-65841) and is supported by the Mayo Clinic College of Medicine's Medical Scientist Training Program. This work was funded by a grant from the National Institutes of Health (NIH), National Institute of Arthritis and Musculoskeletal and Skin Diseases (R01 AR46849). Research reported in this publication was supported by the National Institute of Aging of the NIH under Award Number R01 AG034676 and grant number UL1TR002377 from the National Center for Advancing Translational Sciences (NCATS), a component of the NIH. The content is solely the responsibility of the authors and does not necessarily represent the official views of the NIH.

${ }^{I}$ K.M. Wilton, BS, Medical Scientist Training Program, Mayo Clinic College of Medicine and Science; ${ }^{2}$ S.J. Achenbach, MS, Division of Biomedical Statistics and Informatics, Mayo Clinic College of Medicine and Science; ${ }^{3} J$.M. Davis III, MD, MS, Division of Rheumatology, Mayo Clinic College of Medicine and Science; ${ }^{4}$ E. Myasoedova, MD, PhD, E.L. Matteson, MD, Division of Rheumatology, and Division of Epidemiology, Department of Health Sciences Research, Mayo Clinic College of Medicine and Science; ${ }^{5}$ C.S. Crowson, PhD, Division of Biomedical Statistics and Informatics, and Division of Rheumatology, Mayo Clinic College of Medicine and Science, Rochester, Minnesota, USA.

$J M D$ has received consulting fees and/or honoraria from AbbVie and Sanofi-Genzyme (< $\$ 10,000$ each) and research support from Pfizer. The other authors declare no conflict of interest relevant to this article.

Address correspondence to Dr. C.S. Crowson, Mayo Clinic, 200 1st St. SW, Rochester, MN 55905, USA.Email: Crowson@mayo.edu.

Accepted for publication December 16, 2020.
Rheumatoid arthritis (RA) is a systemic autoimmune inflammatory disease associated with increased mortality. ${ }^{1}$ Cardiovascular (CV) disease is estimated to be responsible for approximately $30-50 \%$ of deaths seen in patients with RA., ${ }^{1,2}$ Compared to the general population, patients with RA have an increased risk of CV disease, including coronary artery disease, myocardial infarction (MI), heart failure (HF), cerebrovascular events, venous thromboembolism, and sudden death. ${ }^{2-7}$ This increased $\mathrm{CV}$ risk in $\mathrm{RA}$ is derived from a combination of traditional $\mathrm{CV}$ risk factors (e.g., smoking, hyperlipidemia, hypertension [HTN], diabetes mellitus [DM], obesity, metabolic syndrome) and increased risk driven by the inflammatory environment. An estimated $30 \%$ of $\mathrm{CV}$ events in RA are secondary to RA disease activity and $49 \%$ are attributable to traditional CV risk factors. ${ }^{8}$

Within the general population, erectile dysfunction (ED) is a "harbinger" of CV disease, developing 2-3 years before CV symptoms and 2-5 years before CV events. ${ }^{9,10,11}$ The majority of $\mathrm{ED}$ is vasogenic in origin secondary to atherosclerosis and endothelial dysfunction within the pelvic vasculature. Given this common mechanism between ED and CV disease, Montorsi, et $a l^{12}$ proposed the "artery size hypothesis": ED presents before $\mathrm{CV}$ symptoms because atherosclerosis deposits occur evenly among blood vessels, affecting small blood vessels such as those in the penis, earlier.

Sexual dysfunction and ED are common both in the general 
population and in men with inflammatory arthritis. In RA, ED prevalence has been reported to be either increased ${ }^{13,14,15}$ or similar ${ }^{16}$ to the general male population. RA severity ${ }^{16,17,18}$ and CV score ${ }^{17}$ have been correlated with sexual dysfunction in RA.

Better understanding of risk factors for $\mathrm{CV}$ events in inflammatory arthritis would help with prediction and prevention. Given the clear correlation of ED with CV risk in the general population, we aimed to define the prevalence of ED in men with RA and to determine if ED is associated with an increased $\mathrm{CV}$ risk in RA.

\section{METHODS}

Study populations. Both cohorts were formed using data from the Rochester Epidemiology Project (REP $)^{19}$ and included individuals who were aged $\geq 18$ years and were residents of Olmsted County (Minnesota, USA). The inception cohort of patients with RA was updated from a previous report. ${ }^{20}$ This cohort contained all men first diagnosed with RA within Olmsted County between January 1, 1980, and December 31, $2007(\mathrm{n}=260)$. The incidence date for men with RA was defined as the first date on which they met at least 4 of the 7 American College of Rheumatology 1987 classification criteria for RA. ${ }^{21}$ A comparison cohort of men with a similar age was created to match the RA cohort and given an index date that corresponded to the incidence date of the respective subject with RA. Longitudinal follow-up for the cohorts was completed until death, migration from Olmsted County, or December 31, 2018. Any identified subjects who did not provide consent for use of their medical records for research purposes were excluded from the study. This study was approved by the institutional review boards at the Mayo Clinic (17-002943) and the Olmsted Medical Center (024-OMC-17), both located in Rochester, Minnesota.

Data abstraction. Relevant data were abstracted from the medical record and used in conjunction with previously extracted data. New data collection included information about ED diagnosis (date of diagnosis, type of $\mathrm{ED}$, diagnosing provider specialty), risk factors for ED (alcohol concerns, depression, anxiety, hypogonadism, thyroid disease, Peyronie disease, penile or spinal trauma, systemic neurologic disease, radical prostatectomy, or pelvic radiation), treatments for ED (phosphodiesterase-5 [PDE5] inhibitors, testosterone, penile self-injection, penile vacuum device use, or surgical penile device placement), and common medications (aspirin, antihypertensives, antidepressants, antiandrogens) that may affect ED. In addition, the number of days in which the patient saw a clinical provider were recorded for 1 year before and after ED diagnosis. Diagnoses of depression or anxiety as well as aspirin use were recorded within 1 year of ED diagnosis. These data were recorded in the secure Research Electronic Data Capture system without any personally identifying information. For the RA and comparator cohorts, data on demographics, $\mathrm{CV}$ risk factors, and events were previously collected as described. ${ }^{20}$

Previous data collection included CV disease and noncardiac vascular events occurring at any time during the patient's history. CV disease events included MI (hospitalized or silent), revascularization procedures, angina, and HF. MI and HF were both defined using objective criteria commonly used in epidemiologic studies, as previously described. ${ }^{22}$ Noncardiac vascular diseases, including venous thromboembolism (deep venous thrombosis or pulmonary embolism), cerebrovascular events (hemorrhagic stroke, nonhemorrhagic stroke, transient ischemic attack, or amaurosis fugax), and peripheral arterial events (abdominal aortic aneurysm, renal artery stenosis, peripheral artery disease, or arterial thromboembolism) were previously collected based on fulfillment of previously defined criteria. ${ }^{23}$ Smoking status and BMI were obtained at RA incidence/index date. HTN, dyslipidemia, and DM were abstracted from the medical records at RA incidence/ index date and throughout follow-up, and were defined using physician diagnosis and standardized diagnostic criteria. ${ }^{22}$

Statistical methods. Descriptive statistics (quantity, percentage, mean, SD, etc.) were used to summarize the data. In the case of continuous numeric data, characteristics of the cohorts were compared by Kruskal-Wallis testing for statistical significance. In the case of categorical groups, chi-square or Fisher exact testing was used as appropriate to determine statistically significant differences. Cumulative incidence of ED in each of the cohorts was completed and adjusted for competing risk of death, ${ }^{24}$ which accounts for those who died before experiencing ED without censoring, and thus avoids overestimating the incidence of ED in surviving members of the cohort. Subjects who were diagnosed with ED before their respective incidence date/index date were excluded from the cumulative incidence analysis. Cox proportional hazard models were used to assess the effect of ED on the subsequent development of $\mathrm{CV}$ events and diagnoses. The interaction between RA/non-RA status and presence of ED was assessed to determine whether ED had a differential effect on CV disease in patients with RA. Subjects who were diagnosed with the $\mathrm{CV}$ event before their respective incidence date/index date were excluded from the Cox models. Analysis was adjusted for age and calendar year at incidence with or without adjustment for DM, HTN, dyslipidemia, obesity, and smoking status. Time-dependent covariates were used for covariates that developed during follow-up (i.e., ED, HTN, DM, and dyslipidemia). Cox models were also used to assess the effect of PDE5 inhibitors (using a time-dependent covariate) in the subset of patients with ED. The analysis of PDE5 started at the RA incidence/index date or the ED date, whichever came later, because patients entered the cohorts at RA incidence for patients with RA and index date for subjects without RA, and they were not at risk of PDE5 use until ED diagnosis. CV outcomes with $<10$ observed events were not analyzed due to lack of statistical power. Person-year methods were used to estimate rates of ED among men aged $<50$ years and men aged $\geq 50$ years. Poisson regression models were used to compare rates of ED within age groups between men with and without RA. Analyses were performed using SAS version 9.4 (SAS Institute) and R 3.6.2 (R Foundation for Statistical Computing).

\section{RESULTS}

The study population included those men in Olmsted County diagnosed with RA ( $\mathrm{n}=260)$ and age-matched male comparators not known to have RA $(\mathrm{n}=260)$. As shown in Table 1 , the comparators had a well-matched age at arthritis incidence/index date. Baseline prevalence of ED at time of arthritis incidence/ index date was similar between men with RA and comparators. At the time of RA incidence/index date, the RA group and comparators had similar baseline CV characteristics (Table 1). There was a trend toward more smoking $(P=0.05)$ and more dyslipidemia $(P=0.05)$ in the men with RA.

Cumulative incidence of ED over time was assessed in relation to the RA incidence/index date. Over the course of this study, men with RA did not have a significantly different rate of ED compared to their age-matched comparators, in either an age-adjusted (HR 0.80; 95\% CI 0.55-1.16) or unadjusted model (HR 0.79; 95\% CI 0.54-1.14; Figure 1). Men aged $<50$ years $(P=0.74$; Table 2$)$ and men aged $\geq 50$ years $(P=0.17$; Table 2$)$ had similar rates of ED diagnosis regardless of RA status.

Potential confounding factors for ED were assessed. These risk factors included clinician suspicion of psychogenic ED or alcohol concerns, use of certain medications (aspirin use, antihypertensives, antidepressants, antiandrogens), medical conditions that can interfere with erectile function (hypogonadism, thyroid disorders, Peyronie disease, neurologic disease, depression, anxiety), relevant past trauma, and iatrogenic causes of ED (radical prostatectomy and radiation therapy). As shown in Table 3, there were no statistically significant increases in 
Table 1. Baseline characteristics of RA and comparator study populations.

\begin{tabular}{lccc}
\hline & $\begin{array}{c}\mathrm{RA}, \\
\mathrm{n}=260\end{array}$ & $\begin{array}{c}\text { Non-RA, } \\
\mathrm{n}=260\end{array}$ & $P$ \\
\hline $\begin{array}{l}\text { Age, yrs, mean (SD) } \\
\text { Follow-up after RA incidence/ } \\
\text { index date, yrs, median (IQR) }\end{array}$ & $57.6(14.4)$ & $57.7(14.4)$ & 0.98 \\
Race, White, $\mathrm{n} / \mathrm{N}(\%)$ & $243 / 256(95)$ & $243 / 254(96)$ & 0.69 \\
BMI at index, kg/m², mean (SD) & $28.3(4.8)$ & $27.9(5.2)$ & 0.38 \\
Obesity, BMI $\geq 30 \mathrm{~kg} / \mathrm{m}^{2}$ & $77(30)$ & $68(26)$ & 0.39 \\
Current smoker & $64(25)$ & $46(18)$ & 0.05 \\
Diabetes & $32(12)$ & $22(8)$ & 0.15 \\
Dyslipidemia & $162(62)$ & $140(54)$ & 0.05 \\
Hypertension & $103(40)$ & $90(35)$ & 0.24 \\
Prior erectile dysfunction & $24(9)$ & $27(10)$ & 0.66 \\
Myocardial infarction & $10(4)$ & $16(6)$ & 0.23 \\
Coronary heart disease & $44(17)$ & $45(17)$ & 0.91 \\
Heart failure & $11(4)$ & $9(3)$ & 0.65 \\
Cerebrovascular disease & $13(5)$ & $11(4)$ & 0.68 \\
Peripheral arterial disease & $9(3)$ & $10(4)$ & 0.82 \\
Venous thromboembolism & $14(5)$ & $8(3)$ & 0.19 \\
Cerebrovascular or peripheral & & & \\
arterial disease & $20(8)$ & $19(7)$ & 0.87 \\
Any cardiovascular disease & $50(19)$ & $53(20)$ & 0.74 \\
\hline
\end{tabular}

Values are expressed as n (\%) unless otherwise indicated. RA: rheumatoid arthritis.

predisposing factors for $\mathrm{ED}$ in the men with $\mathrm{RA}$ in relation to the comparators. There was a decreased incidence of suspected psychogenic ED in men with RA in relation to the comparator cohort $(P=0.04)$. Of note, patients with RA and ED had more medical visits both in the year preceding $(P=0.009)$ and in the year following RA criteria fulfillment $(P=0.03)$, and might be diagnosed with ED by subspecialty providers, although this did not reach statistical significance $(P=0.10)$. Comparators were treated for $\mathrm{ED}$ in a similar manner to the men in the RA cohort. The most common treatment for ED in the first year following diagnosis was PDE5 inhibitors in both cohorts. In total, PDE5 inhibitors were used in more than half of patients with ED in this study. In these cohorts, all other treatments were rarely used to treat $\mathrm{ED}$ within the first year.

The incidence of major $\mathrm{CV}$ events and diagnoses was assessed in both the RA cohort and comparators with relation to ED status. As shown in Table 4, when adjusted for age, calendar year of incidence, DM, HTN, dyslipidemia, obesity, and smoking status, patients with RA who also had ED had an increased risk for peripheral arterial disease (HR 2.22, 95\% CI 0.98-5.03), which did not reach statistical significance, and a decreased risk of MI (HR 0.26, 95\% CI 0.07-0.90), HF (HR 0.49, 95\% CI $0.25-0.94$ ), and all-cause death (HR 0.56, 95\% CI 0.36-0.87). None of these events were significantly increased in comparators with ED. The interaction between RA diagnosis and ED was only statistically significant for an increased risk of peripheral arterial disease $(P=0.02)$, indicating that $\mathrm{ED}$ had a stronger effect on peripheral arterial disease in patients with RA than in comparators. Results were similar when adjusted only for calendar year and age.

The occurrence of $\mathrm{CV}$ events in men with RA and $\mathrm{ED}$ on PDE5 inhibitors was compared to those seen in men with RA and ED not on PDE5 inhibitors. As shown in Table 5, the risk of death overall was decreased in men with RA and ED on PDE5 inhibitors (HR 0.35, 95\% CI 0.16-0.79). Additionally, the risk of coronary heart disease (HR $0.44,95 \%$ CI $0.18-1.12$ ), HF (HR 0.41, 95\% CI 0.13-1.30), and CV disease overall (HR 0.49, 95\% CI 0.20-1.19) were decreased in men on PDE5 inhibitors, but these associations did not reach statistical significance. Interestingly, both ED in men with RA (Table 4) and PDE5 use in men with RA and ED were associated with a decreased risk of death (Table 5). In a similar manner, both presence of

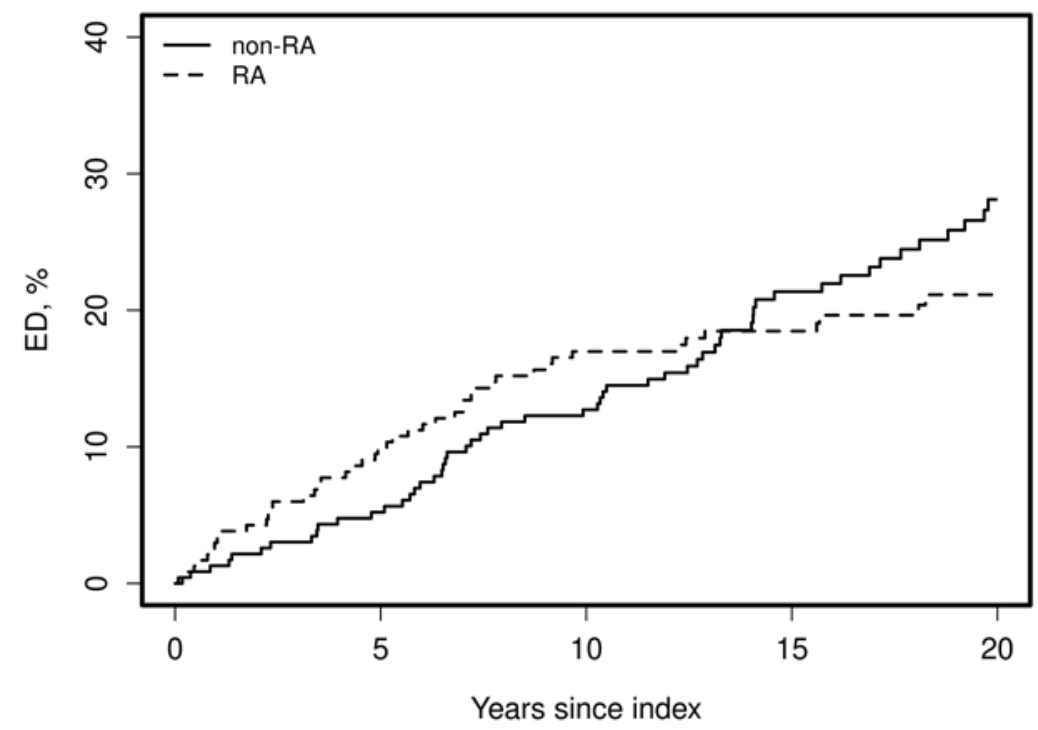

Figure 1. The cumulative incidence of ED was assessed over time among patients with prevalent ED at RA incidence date or index date in non-RA comparators. Age-adjusted analysis comparing the risk of ED in patients with and without RA resulted in an HR 0.80 (95\% CI 0.55-1.16). ED: erectile dysfunction; RA: rheumatoid arthritis. 
Table 2. Association of ED and RA by age group.

\begin{tabular}{lccccc}
\hline Age, Yrs & Patients & ED Diagnoses & PY Counted & Rate per 1000 PY & Rate Ratio (95\% CI) \\
\hline$<50$ & Men with RA & 6 & 650.32 & 9.2 & $1.22(0.37-3.99)$ \\
& Comparators & 5 & 660.36 & 7.6 & 0.74 \\
\multirow{2}{*}{50} & Men with RA & 42 & 2411.14 & 17.4 & $0.76(0.51-1.12)$ \\
& Comparators & 60 & 2613.36 & 23.0 & 0.17 \\
& & & & & \\
\hline
\end{tabular}

ED: erectile dysfunction; PY: person-years; RA: rheumatoid arthritis.

Table 3. Potential diagnostic confounders and initial treatment strategies in men with ED.

\begin{tabular}{|c|c|c|c|}
\hline & $\begin{array}{c}\mathrm{RA} \\
\mathrm{n}=48\end{array}$ & $\begin{array}{c}\text { Non-RA } \\
\text { Comparators, } \\
n=65\end{array}$ & $P$ \\
\hline \multicolumn{4}{|l|}{ Potential confounders } \\
\hline Possible psychogenic ED & $0(0)$ & $6(9)$ & 0.04 \\
\hline Alcohol concerns & $3(6)$ & $5(8)$ & $>0.99$ \\
\hline Antihypertensive use & $23(48)$ & $31(48)$ & 0.98 \\
\hline Aspirin use within $1 \mathrm{yr}$ & $27(56)$ & $36(55)$ & 0.93 \\
\hline Antiandrogen use & $0(0)$ & $1(2)$ & $>0.99$ \\
\hline Antidepressant use & $7(15)$ & $9(14)$ & 0.91 \\
\hline Depression within $1 \mathrm{yr}$ & $6(13)$ & $10(15)$ & 0.66 \\
\hline Anxiety within $1 \mathrm{yr}$ & $3(6)$ & $6(9)$ & 0.56 \\
\hline Hypogonadism diagnosis & $4(8)$ & $2(3)$ & 0.40 \\
\hline Thyroid disease diagnosis & $3(6)$ & $6(9)$ & 0.73 \\
\hline Peyronie disease & $1(2)$ & $1(2)$ & $>0.99$ \\
\hline Penile trauma & $0(0)$ & $0(0)$ & - \\
\hline Spinal cord trauma & $0(0)$ & $1(2)$ & $>0.99$ \\
\hline Neurological disease & $4(8)$ & $5(8)$ & $>0.99$ \\
\hline Radical prostatectomy & $4(8)$ & $4(6)$ & 0.72 \\
\hline Radiation therapy & $0(0)$ & $1(2)$ & $>0.99$ \\
\hline \multicolumn{4}{|l|}{$\begin{array}{l}\text { Days with outpatient visits or } \\
\text { hospitalization, mean (SD) }\end{array}$} \\
\hline Preceding year & $7.0(6.9)$ & $4.1(4.0)$ & 0.009 \\
\hline Following year & $9.0(9.4)$ & $6.0(5.4)$ & 0.03 \\
\hline Diagnosing physician & & & 0.10 \\
\hline Family practice & $13(27)$ & $14(22)$ & \\
\hline Internal medicine & $22(46)$ & $40(62)$ & \\
\hline Urology & $4(8)$ & $7(11)$ & \\
\hline Subspecialty internal medicine & $9(19)$ & $3(5)$ & \\
\hline Other & $0(0)$ & $1(2)$ & \\
\hline \multicolumn{4}{|l|}{ Treatments in first year } \\
\hline Phosphodiesterase-5 inhibitor & $27(56)$ & $41(63)$ & 0.46 \\
\hline Testosterone replacement & $4(8)$ & $1(2)$ & 0.16 \\
\hline Penile self-injection & $4(8)$ & $2(3)$ & 0.40 \\
\hline Penile vacuum device & $2(4)$ & $1(2)$ & 0.57 \\
\hline Penile device placement & $1(2)$ & $0(0)$ & 0.42 \\
\hline
\end{tabular}

Values are expressed as $\mathrm{n}(\%)$ unless otherwise indicated. ED: erectile dysfunction; RA: rheumatoid arthritis.

ED (HR 0.49, 95\% CI 0.25-0.94) and use of PDE5 inhibitors (HR 0.41,95\% CI 0.13-1.30) were associated with decreased risk of HF in men with RA. Conversely, men with RA and ED experienced an increased risk of peripheral arterial disease (HR 2.22; 95\% CI 0.98-5.03) that did not reach statistical significance, but PDE5 inhibitor use showed no association with peripheral arterial disease (HR 1.00, 95\% CI 0.28-3.53; Table 5).

\section{DISCUSSION}

In this study, ED incidence was not increased in men with RA and ED diagnosis was not associated with an increased risk of $\mathrm{CV}$ disease in RA. There was an increased risk of peripheral arterial disease that was dependent on both ED and RA diagnosis, but this association did not reach statistical significance. Additionally, ED was associated with a decreased risk of MI, HF, and mortality in men with RA. Interestingly, use of PDE5 inhibitors in men with RA and ED was associated with a decreased risk of death and a trend toward decreased rate of some CV events and diagnoses.

The prevalence of ED in RA has been reported as increased ${ }^{13,14,15,25}$ or similar ${ }^{16}$ to rates of ED in controls. In this study, we did not observe an increased rate of ED in men with RA. This could be secondary to well-controlled RA disease activity, as inflammatory disease activity increases ED prevalence. ${ }^{16,17,18}$ Additionally, this result could be confounded by variable follow-up, as men with RA had more frequent medical visits. Medication-induced ED may be a confounding factor, as sexual dysfunction in men associated with methotrexate use has been described. ${ }^{26,27}$ In addition, potential age dependence to ED risk in RA could explain heterogeneous study results. ${ }^{13,14,15,16,25}$ Overall, this study does not support increased ED incidence in men with RA, but confounding cannot be excluded.

Due to the association of $\mathrm{ED}$ with $\mathrm{CV}$ events in the general population, we hypothesized that men with RA would have an increased rate of most $\mathrm{CV}$ events when also diagnosed with $\mathrm{ED}$. Interestingly, this was not the case. Most CV events were not statistically associated with ED diagnosis, potentially indicating that risk from ED and RA are redundant. Peripheral arterial disease was the only $\mathrm{CV}$ condition that was increased by the presence of ED, with potential interaction between RA and ED diagnosis. Both ED and peripheral arterial disease are secondary to endothelial dysfunction in the lower body, and reduced blood flow secondary to atherosclerosis and decreased vasodilation. These similarities may link these conditions more closely than other $\mathrm{CV}$ conditions.

In this study, ED in RA was associated with a decreased risk of MI, HF, and overall mortality. This finding is of interest given the importance of these events and the initial hypothesis that $\mathrm{ED}$ diagnosis would be associated with an increased risk of these conditions. Although it would seem that more frequent follow-up in men with RA could lead to increased access to CV preventive measures, previous studies have shown that patients with RA have suboptimal $\mathrm{CV}$ prevention. ${ }^{28,29,30} \mathrm{CV}$ risk may also be confounded as the men who are diagnosed with ED may have 
Table 4. Association between erectile dysfunction and cardiovascular outcomes in patients with RA and comparator cohort, adjusted for age, calendar year, diabetes mellitus, hypertension, dyslipidemia, obesity, and smoking status.

\begin{tabular}{|c|c|c|c|c|c|}
\hline \multirow[b]{2}{*}{ CV Outcome } & \multicolumn{2}{|c|}{ RA } & \multicolumn{2}{|c|}{ Comparators } & \multirow[b]{2}{*}{ Interaction $P$} \\
\hline & CV Events/Total & $\operatorname{HR}(95 \% \mathrm{CI})$ & CV Events/Total & $\mathrm{HR}(95 \% \mathrm{CI})$ & \\
\hline Myocardial infarction & $29 / 250$ & $0.26(0.07-0.90)$ & $21 / 244$ & $1.11(0.41-3.03)$ & 0.11 \\
\hline Heart failure & $61 / 249$ & $0.49(0.25-0.94)$ & $42 / 251$ & $0.84(0.41-1.74)$ & 0.20 \\
\hline Cerebrovascular disease & $38 / 247$ & $0.98(0.46-2.11)$ & $33 / 249$ & $0.59(0.25-1.41)$ & 0.24 \\
\hline Peripheral arterial disease & $29 / 251$ & $2.22(0.98-5.03)$ & $21 / 250$ & $0.46(0.15-1.40)$ & 0.02 \\
\hline Cerebrovascular/peripheral arterial disease & $54 / 240$ & $1.62(0.86-3.05)$ & $41 / 241$ & $0.57(0.26-1.24)$ & 0.06 \\
\hline Any $\mathrm{CV}$ disease & $104 / 210$ & $1.15(0.68-1.97)$ & $73 / 207$ & $1.05(0.61-1.83)$ & 0.63 \\
\hline Death & $130 / 260$ & $0.56(0.36-0.87)$ & $104 / 259$ & $0.94(0.58-1.52)$ & 0.24 \\
\hline
\end{tabular}

CV: cardiovascular; RA: rheumatoid arthritis.

Table 5. Association between phosphodiesterase -5 inhibitor use and CV events in erectile dysfunction patients with RA and comparator cohorts, adjusted for age.

\begin{tabular}{lcccc}
\hline & & RA & \multicolumn{2}{c}{ Comparators } \\
CV Outcome* & CV Events/Total & HR (95\% CI) & CV Events/Total & HR (95\% CI) \\
\hline Myocardial infarction & $3 / 66$ & - & $6 / 80$ & - \\
Coronary heart disease & $21 / 53$ & $0.44(0.18-1.12)$ & $19 / 70$ & $0.93(0.30-2.87)$ \\
Heart failure & $14 / 69$ & $0.41(0.13-1.30)$ & $12 / 84$ & $0.55(0.17-1.76)$ \\
Cerebrovascular disease & $14 / 64$ & $1.89(0.45-7.98)$ & $7 / 81$ & - \\
Peripheral arterial disease & $15 / 67$ & $1.00(0.28-3.53)$ & $4 / 85$ & - \\
Venous thromboembolism & $7 / 62$ & - & $7 / 83$ & - \\
Cerebrovascular/peripheral arterial disease & $22 / 61$ & $0.91(0.34-2.43)$ & $9 / 80$ & - \\
Any CV disease & $24 / 47$ & $0.49(0.20-1.19)$ & $21 / 65$ & $0.76(0.27-2.14)$ \\
Death & $31 / 72$ & $0.35(0.16-0.79)$ & $27 / 87$ & $0.84(0.37-1.91)$ \\
\hline
\end{tabular}

${ }^{*}$ Analyses were only performed for outcomes with at least 10 events. CV: cardiovascular; RA: rheumatoid arthritis.

variation in health-based limitations for sexual activity, capacity for self-care, independence, and education concerning sexual health. Another less likely possibility is that vasoactive medications, such as PDE5 inhibitors, which are used for ED, may have additional CV benefits in this population.

Given that a large number of the men with ED were prescribed PDE5 inhibitors, and that PDE5 inhibitors have been noted to be associated with a cardioprotective effect in the general population, ${ }^{24,25}$ we hypothesized that the use of PDE5 inhibitors may correlate with a decreased risk of some $\mathrm{CV}$ events in the men with RA. Specifically, in this study, there was a decreased risk of death in men with RA and ED on PDE5 inhibitors vs men with RA and ED not on PDE5 inhibitors. There were also decreased risks of several CV conditions that did not reach statistical significance, likely secondary to small population size. This decreased $\mathrm{CV}$ risk in men with RA and ED on PDE5 inhibitors may not be directly related to the pharmacologic action of the medication. Specifically, prescription and use of PDE5 inhibitors is confounded by socioeconomic factors, insurance coverage, and education. Further, like ED diagnosis itself, prescription for PDE5 inhibitors may be confounded by the level of patient selfcare and sexual health education, as patients usually initiate the conversation. Additionally, in this study, prescription of PDE5 inhibitors was used; we were not able to measure how often men were taking these medications, which are usually taken on an as-desired/needed basis. In some cases, the men may have been prescribed the medication and never used it. Therefore, the difference in $\mathrm{CV}$ events seen in this study could actually be a reflection of prescribing patterns of physicians; specifically, physicians are less likely to prescribe a PDE5 inhibitor to men with complex and unstable health conditions because these men are presumably less likely to request the medications, may not be healthy enough for sexual activity, or may be reluctant to take the medications given their well-known interactions with nitrates. In addition, health literacy may confound which men are prescribed PDE5 inhibitors.

Although a number of potential confounders may affect the estimates of CV disease, this result is intriguing. Specifically, in the general population the association between ED and CV risk virtually disappeared after sildenafil was first introduced onto the market for ED. ${ }^{31,32}$ Since then, PDE5 inhibitors have been studied for use in various types of HF and ischemia (reviewed previously ${ }^{33}$ ). PDE5 inhibitors have been shown to prevent cardiac remodeling, ${ }^{34,35,36}$ decrease cardiomyocyte apoptosis and necrosis, ${ }^{35,37}$ and attenuate symptoms of HF. ${ }^{36}$ These cardioprotective effects of PDE5 inhibitors may possibly contribute to the 
decreased rate of MI and HF observed in this cohort. Of note, in this cohort, PDE5 inhibitors were used for ED, and thus were likely used intermittently. This intermittent use of medications may either indicate that PDE5 inhibitors are effective even when used sporadically in this high-risk population or, more likely, that they are not directly responsible for the decreased incidence of $\mathrm{MI}$ and death seen in this cohort. In either case, at least in this study, ED and PDE5 inhibitor use was not associated with an increased risk of $\mathrm{CV}$ events or death.

To our knowledge, this study is the first to define the CV risk associated with $\mathrm{ED}$ in patients with $\mathrm{RA}$, and to specifically define the population-based incidence of ED in men with RA. Strengths of the study include the completeness of the medical data based upon the REP, ${ }^{19}$ which is a geographically defined population where medical records for the entire population of Olmsted County, Minnesota are available. This allows for a population-based assessment, including all consenting individuals without internal bias. In addition, access to the medical record allows for confirmation of International Classification of Diseases (ICD) code-based physician diagnosis and extraction of details not traditionally encapsulated in ICD code or insurance-based analysis. The REP system allows for long-term longitudinal follow-up over decades, as well as extraction of all known healthcare data from sites within the county. In addition, age-matched male comparators were able to be selected from the general population, allowing for a geographically based comparator cohort. Limitations to this study include the demographic composition of the population studied; the population included in the REP reflects a county that is disproportionately White, which may limit generalizability. In addition, this study was retrospective, including only that information encapsulated in the medical record (availability bias), and thus is subject to all of the inherent limitations of the study design. Additionally, this study was limited to the size of Olmsted County, which led to a relatively small overall sample size, which limited our ability to detect differences in subanalysis. Another limitation is the frequent underdiagnosis of ED..$^{38}$ In our present study, only those men with documented ED were included; men with subclinical ED, penile atherosclerosis, or without concern about their ED were excluded. Prospective studies would ascertain a more accurate ED risk. Additionally, RA research can be affected by index event bias, ${ }^{39}$ which often presents as a risk factor paradox, where risk factors are not consistent among studies or are not logical.

Future studies on the implications of ED on CV risk in RA may help to inform the assessment and treatment of $\mathrm{CV}$ risk in inflammatory arthritis. First, assessment of whether ED is a reflection of traditional CV risk or is altered by the inflammatory state could help to inform risk-based models. It would be important to determine whether the increased incidence of peripheral arterial disease among in men with ED and RA is related to disease activity, and why peripheral arterial disease differs from other CV events. Perhaps most intriguing is the apparent decreased risk of MI, HF, and mortality among men with RA who have ED, warranting further study.

The incidence of ED in patients with RA is similar to that of age-matched comparators. Although ED did not affect the overall rate of $\mathrm{CV}$ events in RA, it was associated with an increased risk of peripheral arterial disease and a decreased risk of MI and HF. While these trends require further study, they may be explained by common pathologic mechanisms, healthcare-related confounding variables, or the use of vasoactive pharmacologic therapy for ED.

\section{REFERENCES}

1. Van den Hoek J, Boshuizen H, Roorda L, Tijhuis G, Nurmohamed M, van den Bos G, et al. Mortality in patients with rheumatoid arthritis: a 15-year prospective cohort study. Rheumatol Int 2017;37:487-93

2. Aviña-Zubieta JA, Choi HK, Sadatsafavi M, Etminan M, Esdaile JM, Lacaille D. Risk of cardiovascular mortality in patients with rheumatoid arthritis: a meta-analysis of observational studies. Arthritis Rheum 2008;59:1690-7.

3. Aviña-Zubieta JA, Thomas J, Sadatsafavi M, Lehman AJ, Lacaille D. Risk of incident cardiovascular events in patients with rheumatoid arthritis: a meta-analysis of observational studies. Ann Rheum Dis 2012;71:1524-9.

4. Solomon DH, Goodson NJ, Katz JN, Weinblatt ME, Avorn J, Setoguchi S, et al. Patterns of cardiovascular risk in rheumatoid arthritis. Ann Rheum Dis 2006;65:1608-12.

5. Maradit-Kremers H, Crowson CS, Nicola PJ, Ballman KV, Roger VL, Jacobsen SJ, et al. Increased unrecognized coronary heart disease and sudden deaths in rheumatoid arthritis: a population-based cohort study. Arthritis Rheum 2005;52:402-11.

6. Gabriel SE. Cardiovascular morbidity and mortality in rheumatoid arthritis. Am J Med 2008;10 Suppl 1:S9-14.

7. Ahlers MJ, Lowery BD, Farber-Eger E, Wang TJ, Bradham W, Ormseth MJ, et al. Heart failure risk associated with rheumatoid arthritis-related chronic inflammation. J Am Heart Assoc 2020:e014661.

8. Crowson CS, Rollefstad S, Ikdahl E, Kitas GD, van Riel P, Gabriel SE, et al; A Trans-Atlantic Cardiovascular Consortium for Rheumatoid Arthritis (ATACC-RA). Impact of risk factors associated with cardiovascular outcomes in patients with rheumatoid arthritis. Ann Rheum Dis 2018;77:48-54.

9. Montorsi F, Briganti A, Salonia A, Rigatti P, Margonato A, Macchi A, et al. Erectile dysfunction prevalence, time of onset and association with risk factors in 300 consecutive patients with acute chest pain and angiographically documented coronary artery disease. Eur Urol 2003;44:360-5.

10. Montorsi P, Ravagnani PM, Galli S, Rotatori F, Veglia F, Briganti A, et al. Association between erectile dysfunction and coronary artery disease. Role of coronary clinical presentation and extent of coronary vessels involvement: the COBRA trial. Eur Heart J 2006;27:2632-9.

11. Baumhäkel M, Böhm M. Erectile dysfunction correlates with left ventricular function and precedes cardiovascular events in cardiovascular high-risk patients. Int J Clin Pract 2007;61:361-6.

12. Montorsi P, Ravagnani PM, Galli S, Rotatori F, Briganti A, Salonia A, et al. The artery size hypothesis: a macrovascular link between erectile dysfunction and coronary artery disease. Am J Cardiol 2005;96:19M-23M.

13. Elst P, Sybesma T, van der Stadt RJ, Prins AP, Muller WH, den Butter A. Sexual problems in rheumatoid arthritis and ankylosing spondylitis. Arthritis Rheum 1984;27:217-20.

14. Nasr MM, El-Shafey AM. Sexual performance in rheumatoid arthritis patients - an unnoticed problem. Egypt Rheumatol 2013;35:201-5. 
15. Blake DJ, Maisiak R, Alarcon GS, Holley HL, Brown S. Sexual quality-of-life of patients with arthritis compared to arthritis-free controls. J Rheumatol 1987;14:570-6.

16. Gaber W, Moghazy A, Niazy M, Salem HK. Risk factors for sexual dysfunction in Egyptian patients with rheumatoid arthritis and its relation to disease activity. Egypt Rheumatol 2017;39:135-8.

17. El Miedany Y, El Gaafary M, El Aroussy N, Youssef S, Ahmed I. Sexual dysfunction in rheumatoid arthritis patients: arthritis and beyond. Clin Rheumatol 2012;31:601-6.

18. Perez-Garcia LF, Te Winkel B, Carrizales JP, Bramer W, Vorstenbosch S, van Puijenbroek E, et al. Sexual function and reproduction can be impaired in men with rheumatic diseases: a systematic review. Semin Arthritis Rheum 2020;50:557-73.

19. St Sauver JL, Grossardt BR, Leibson CL, Yawn BP, Melton LJ 3rd, Rocca WA. Generalizability of epidemiological findings and public health decisions: an illustration from the Rochester Epidemiology Project. Mayo Clin Proc 2012;87:151-60.

20. Wilton KM, Matteson EL, Crowson CS. Risk of obstructive sleep apnea and its association with cardiovascular and noncardiac vascular risk in patients with rheumatoid arthritis: a population-based study. J Rheumatol 2018;45:45-52.

21. Arnett FC, Edworthy SM, Bloch DA, McShane DJ, Fries JF, Cooper NS, et al. The American Rheumatism Association 1987 revised criteria for the classification of rheumatoid arthritis. Arthritis Rheum 1988;31:315-24.

22. Myasoedova E, Crowson CS, Nicola PJ, Maradit-Kremers H, Davis JM 3rd, Roger VL, et al. The influence of rheumatoid arthritis disease characteristics on heart failure. J Rheumatol 2011;38:1601-6.

23. Bacani AK, Gabriel SE, Crowson CS, Heit JA, Matteson EL. Noncardiac vascular disease in rheumatoid arthritis: increase in venous thromboembolic events? Arthritis Rheum 2012;64:53-61.

24. Gooley TA, Leisenring W, Crowley J, Storer BE. Estimation of failure probabilities in the presence of competing risks: new representations of old estimators. Stat Med 1999;18:695-706.

25. Keller JJ, Lin HC. A population-based study on the association between rheumatoid arthritis and erectile dysfunction. Ann Rheum Dis 2012;71:1102-3.

26. Blackburn WD Jr, Alarcon GS. Impotence in three rheumatoid arthritis patients treated with methotrexate. Arthritis Rheum 1989;32:1341-2.

27. Thomas E, Koumouvi K, Blotman F. Impotence in a patient with rheumatoid arthritis treated with methotrexate. J Rheumatol 2000;27:1821-2.

28. Heslinga M, Van Den Oever I, Jonker DL, Griep EN, Griep-Wentink H, Smulders YM, et al. Suboptimal cardiovascular risk management in rheumatoid arthritis patients despite an explicit cardiovascular risk screening programme. Scand J Rheumatol 2019;48:345-52.

29. Schmidt TJ, Aviña-Zubieta JA, Sayre EC, Abrahamowicz M, Esdaile JM, Lacaille D. Quality of care for cardiovascular disease prevention in rheumatoid arthritis: compliance with hyperlipidemia screening guidelines. Rheumatology 2018;57:1789-94.

30. van den Oever IAM, Heslinga M, Griep EN, Griep-Wentink HRM, Schotsman R, Cambach W, et al. Cardiovascular risk management in rheumatoid arthritis patients still suboptimal: the Implementation of Cardiovascular Risk Management in Rheumatoid Arthritis project. Rheumatology 2017;56:1472-8.

31. Frantzen J, Speel TG, Kiemeney LA, Meuleman EJ. Cardiovascular risk among men seeking help for erectile dysfunction. Ann Epidemiol 2006;16:85-90.

32. Dong JY, Zhang YH, Qin LQ. Erectile dysfunction and risk of cardiovascular disease: meta-analysis of prospective cohort studies. J Am Coll Cardiol 2011;58:1378-85.

33. Tzoumas N, Farrah TE, Dhaun N, Webb DJ. Established and emerging therapeutic uses of PDE type 5 inhibitors in cardiovascular disease. Br J Pharmacol 2019;177:5467-88.

34. Giannetta E, Feola T, Gianfrilli D, Pofi R, Dall'Armi V, Badagliacca $\mathrm{R}$, et al. Is chronic inhibition of phosphodiesterase type 5 cardioprotective and safe? A meta-analysis of randomized controlled trials. BMC Med 2014;12:185.

35. Chau VQ, Salloum FN, Hoke NN, Abbate A, Kukreja RC. Mitigation of the progression of heart failure with sildenafil involves inhibition of RhoA/Rho-kinase pathway. Am J Physiol Heart Circ Physiol 2011;300:H2272-9.

36. Guazzi M, Vicenzi M, Arena R, Guazzi MD. PDE5 inhibition with sildenafil improves left ventricular diastolic function, cardiac geometry, and clinical status in patients with stable systolic heart failure: results of a 1-year, prospective, randomized, placebo-controlled study. Circ Heart Fail 2011;4:8-17.

37. Das A, Xi L, Kukreja RC. Phosphodiesterase-5 inhibitor sildenafil preconditions adult cardiac myocytes against necrosis and apoptosis. Essential role of nitric oxide signaling. J Biol Chem 2005;280:12944-55.

38. Jannini EA, Sternbach N, Limoncin E, Ciocca G, Gravina GL, Tripodi F, et al. Health-related characteristics and unmet needs of men with erectile dysfunction: a survey in five European countries. J Sex Med 2014;11:40-50.

39. Choi HK, Nguyen US, Niu J, Danaei G, Zhang Y. Selection bias in rheumatic disease research. Nat Rev Rheumatol 2014;10:403-12. 